\title{
A comparison of the short-term memory performances of pigeons and jackdaws
}

\author{
B. WILSON and R. A. BOAKES \\ University of Sussex, Brighton, England
}

\begin{abstract}
Two experiments employed a delayed conditional discrimination procedure in which half the trials began with the presentation of food and half with no food; following a retention interval, subjects were presented with a choice between red and green keys, a response to one of which was reinforced according to whether the trial had started with food or no food. In Experiment 1, after 38 training sessions during which the retention interval was gradually increased, pigeons performed at a moderate level with intervals of 5 to $7.5 \mathrm{sec}$. A final test produced a steep forgetting function for food trials, but not for no-food trials; performance was unaffected by the duration of the intertrial interval ( 10 or $40 \mathrm{sec}$ ). Experiment 2 used the delayed conditional discrimination procedure to compare short-term memory in jackdaws (Corvus monedulus) with that in pigeons. Although the performance of the jackdaws was below that of the pigeons at the start of training, they showed more rapid learning over long delays, and, in the final test, a shallower forgetting function for food trials than that shown by pigeons. The results suggested superior short-term memory in jackdaws, which may help to explain the better performance of corvids in general when compared with that of pigeons in certain complex learning tasks.
\end{abstract}

It is widely agreed that proficient performance by an animal on certain complex learning tasks involves the employment of a win-stay/lose-shift strategy (Levine, 1959; for a recent review, see Macphail, 1982). In other words, the behavior of the animal comes under the control of the most recent trial and is relatively free from the influence of events preceding that trial. Some of the strongest evidence for the important role of this strategy comes from studies that have found positive transfer from training on a serial-reversal task to the acquisition of learning-set performance, a result first obtained in primates (Schrier, 1974; Schusterman, 1962, 1964; Warren, 1966). In a comparable study using birds, Kamil, Jones, Pietrewicz, and Mauldin (1977) first trained a group of blue jays on a serial-reversal task involving a single pair of objects and then transferred them to an object-discrimination learning set. This group displayed much more rapid acquisition of learning-set performance than a control group initially trained on a single discrimination problem. The differences between the groups were most marked on problems in which animals made an error on the first trial; this provides strong support for the suggestion that the control animals needed to acquire the "lose-shift" part of the strategy in order to perform well.

If good learning-set performance depends on the use of transient memories of what happened on the previous trial, then the interval between trials should be a critical factor. Confirmation of this prediction has been obtained in experiments that have found a large impairment of per-

This research was supported by a grant from the Science and Engineering Research Council, United Kingdom. Requests for reprints should be sent to R. A. Boakes, Laboratory of Experimental Psychology, University of Sussex, Brighton BN1 9QG, England. formance as a result of increasing intertrial intervals from a training value of around $10 \mathrm{sec}$ to values of $2 \mathrm{~min}$ or more; this has been found with both primates (Bessemer \& Stollnitz, 1971) and blue jays (Kamil, Lougee, \& Shulman, 1973; Kamil \& Mauldin, 1975). A further prediction is that species that are good at acquiring learning sets should be those that possess good short-term memories. It was this prediction that the experiments reported here set out to test.

Direct comparisons of the learning-set abilities of pigeons and corvids (Wilson, 1978) not only found faster acquisition in corvids, but also found that they were more sensitive to increases in intertrial interval. Wilson (1978) also compared rooks and pigeons on the serial reversal of a simultaneous vertical-horizontal discrimination; over five reversals, the rooks showed rapid improvement, but the pigeons did not. The unimpressive performance of pigeons in Wilson's comparative research, and in an earlier experiment on learning-set formation (Zeigler, 1961), might be explained by pigeons' relatively poor ability to respond on the basis of events that occurred more than a few seconds previously. The results from a large number of recent studies of short-term memory in the pigeon (e.g., Honig, 1978; Maki, 1981) suggest that this may be the case. However, there does not appear to have been any comparative study of short-term memory in birds, let alone one that examined differences between species known to perform well in learning-set tasks and those found to perform poorly.

Wilson (1978) employed crows and rooks in her learning-set experiments; no animals of these species were readily available for the present study, and jackdaws (Corvus monedulus) were chosen instead as a representative corvid species. We have found this species to be 
less variable than other corvids when studied with Skinner box methods. Possibly, as hole-nesting birds, jackdaws are better able to tolerate confinement in a small space. They have been found to perform at least as well on various complex learning tasks as any other corvid that has been studied (Mackintosh, Wilson, \& Boakes, 1985; Wilson, Mackintosh, \& Boakes, 1985).

\section{EXPERIMENT 1}

The procedure adopted in the present study was the delayed conditional discrimination (DCD) procedure used extensively in recent years to study short-term retention in pigeons (e.g., Colwill \& Dickinson, 1980a, 1980b; Maki, Moe, \& Bierley, 1977). Half of the trials began with the unsignaled presentation of food and the other half without any particular event. After a retention interval on each trial, two choice keys were illuminated, one red and the other green. A response to one color was immediately reinforced on trials beginning with food and a response to the other color was reinforced on no-food trials. Thus, to make a correct choice, a subject had to remember whether food had been presented or not at the onset of the trial some seconds earlier. Before committing the few jackdaws available for the study, we assessed the effectiveness of various parameters of this procedure in Experiment 1, using only pigeons. Because crossspecies comparisons are notoriously open to the objection that the outcome may reflect the use of a particular combination of procedural variables, this experiment examined the possible effect of one such variable, the in tertrial interval, which has been found in other studies to affect the performance of pigeons on this task (e.g., Maki et al., 1977).

\section{Method}

Subjects. The subjects were 6 male homing pigeons, bred in the Universiy of Sussex colony. They had previously served in two short experiments, one on autoshaping and one on simple alternation. They were approximately 2 years old at the start of the experiment and were assigned to two groups of 3 birds in such a way as to counterbalance previous experience. All subjects were housed individually with water freely available, but were deprived of food so as to maintain their weights at $80 \%$ of their immediately prior free-feeding value.

Apparatus. The conditioning chamber was of standard dimensions for pigeons, $30 \times 30 \times 30 \mathrm{~cm}$. One wall served as an intelligence panel, containing three square response keys, each $4 \times 4 \mathrm{~cm}$, mounted with their bottom edges at a height of $16 \mathrm{~cm}$ above the floor. The two side keys were separated from the center key by an edge-to-edge distance of $3.5 \mathrm{~cm}$. The center key could be transilluminated by white light from a 6-W, 24-V MES bulb mounted directly behind it; each side key could be illuminated by red or green light from identical MES bulbs with standard plastic covers. Reward consisted of 45-mg Noyes pigeon pellets, delivered from a dispenser mounted behind the intelligence panel into a recessed food tray that could be lit from above by white light from an MES bulb. The square central aperture providing access to this tray measured $6 \times 6 \mathrm{~cm}$, with its bottom edge at a height of $7 \mathrm{~cm}$ above the chamber floor. The chamber could be illuminated by dim white light from the houselight, an MES bulb mounted centrally at the top of the rear wall.
The chamber was enclosed within a light-tight and soundattenuating chest. Ventilating fans and white noise from a speaker on the rear wall of the chamber provided auditory masking throughout each session. Control of events and recording was by means of electromechanical equipment.

Procedure. Each experimental session contained 60 trials. The intertrial interval was always $40 \mathrm{sec}$ for the Long group and $10 \mathrm{sec}$ for the Short group.

For the first 10 sessions, a no-delay procedure was used. Each trial began with 4-sec illumination of the center key with white light; the offset of this light was followed by a 3-sec interval. On half the trials (food trials), this interval began with the delivery of two food pellets with an interval of $0.5 \mathrm{sec}$ between them; the foodtray was illuminated for the whole $3 \mathrm{sec}$. On the other trials (no-food trials), no pellets were delivered and the tray was not illuminated. At the end of the 3-sec interval the two side keys were lit, one with red and the other with green. For half the birds on food trials, a single response to the red key was followed immediately by offset of the keylights, the delivery of two food pellets, and the onset of 3 -sec tray illumination, but a response to the green key simply extinguished the keylights and produced a 3-sec blackout period. Similarly, for half the birds on no-food trials a first response to the green key produced reinforcement, but a first response to the red key produced a 3-sec blackout period. In either case, the keylights were immediately extinguished and no further response to either side key had any effect until the next trial. For the remaining subjects in each group the opposite assignment of the two colors was used.

In all subsequent phases of the experiment, the houselight was on only during the intertrial interval and the initial $4 \mathrm{sec}$ of each trial when the center key was lit. However, since initially some of the birds were very slow to peck the side keys in the absence of a houselight, this was left on throughout some of the early sessions in this phase.

The procedure generated four kinds of trial: food with red on left, food with red on right, no-food with red on left, and no-food with red on right. Each kind of trial occurred 15 times within each session in a semirandom sequence, with the constraint that no kind of trial could occur more than three times in succession. Five such trial sequences were programmed on punched tape and the tapes were changed in varying sequence from session to session throughout the experiment.

Following the 10 sessions of the no-delay phase, the procedure was modified by introducing an interval of $t \mathrm{sec}$ between the end of the 3-sec food/no-food period and the onset of the side keylights. During this retention interval the chamber was completely dark. The initial value of $t$ was $0.5 \mathrm{sec}$, and over a total of 38 sessions its value was adjusted in the following manner. A series of intervals was defined in which each value was approximately 1.5 times the value of its predecessor, taken to the nearest $0.5 \mathrm{sec}$. The intervals were $0.5,1.0,1.5,2.5,3.5,5.0,7.5,11.5 \mathrm{sec}$ and so on. When a subject performed at a level of 40 or more correct trials out of 60 for two successive sessions, the retention interval for the next session was set at the next value. When, in a single session, the number of correct trials was less than 40 , the interval for the next session was reduced to the value immediately below.

After the end of this training with adjusted values of retention interval, subjects were given two further sessions, in which the value of $t$ was set to the median value of the settings for that subject over the previous five sessions. This was to minimize the possibility that immediately prior to the final tests some subjects might have performed badly.

The final phase of the experiment contained two test sessions in which, for the first time, retention intervals were changed from trial to trial. Apart from this, conditions were the same as training conditions. Six intervals were used: 2, 6, 10, 14, 18, and $22 \mathrm{sec}$. Each interval occurred 10 times within each test session; over both sessions, each interval occurred 5 times with each of the four kinds of trial. The sequences of intervals were otherwise random, but with the added constraint that the same interval could not occur more than twice in succession. 
During training sessions, the total number of center-key responses during the initial $4 \mathrm{sec}$ of each trial, the number of correct food trials, the number of correct no-food trials, and the number of responses to the right key were recorded for each session. During the final two test sessions, trial-by-trial records of these measures were made.

\section{Results and Discussion}

Results from the training phase of the experiment are given in the left-hand panel of Figure 1, which shows the median $t$ settings over successive blocks of 5 sessions for the last 35 sessions of the training procedure. These values have been averaged separately for the 3 Long and the 3 Short subjects. As the figure suggests, there was no indication that the duration of the intertrial interval affected acquisition. Over the last 5 training sessions, the median $t$ settings for the Short subjects were 5, 7.5, and $11.5 \mathrm{sec}$, while those for the Long subjects were 5,5 , and $11.5 \mathrm{sec}$. On only one occasion did a subject reach a score of 40 or more correct when the value of the retention interval was increased from $11.5 \mathrm{sec}$ to the next setting of $17 \mathrm{sec}$.

Performance during the final test sessions is shown in the right-hand panel of Figure 1. As in acquisition, no sign of any difference between the two groups of subjects appeared in the results from these sessions; therefore, data for all 6 subjects have been pooled in this figure. The median percentage of correct trials for each retention inter$\mathrm{val}$ is shown. The trend toward lower performance at longer intervals was found to be significant [Page's L $(6,6)$ $=517, p<.01]$. Also shown in Figure 1 are the separate results for food and no-food trials. It may be seen that the percentage of correct responses on food trials decreased rapidly as the retention interval increased, whereas performance on no-food trials remained unaffected. Nonparametric trend analysis confirmed this impression: whereas no trend was detected for no-food trials, a reliable trend was obtained on food trials $[L(6,6)=533$, $\mathrm{p}<.01]$.

The absence of an intertrial spacing effect failed to confirm the result reported by Maki et al. (1977, Experiment 2). This discrepancy may reflect Maki et al.'s use of a within-subjects comparison, their use of a different range of intervals, or the extensive training they gave their 3 subjects on the longer interval $(20 \mathrm{sec})$ before introducing the shorter one $(2 \mathrm{sec})$. For our purposes, the failure to detect an intertrial effect meant that any difference between pigeons and jackdaws to be found in Experiment 2 was unlikely to be crucially influenced by the choice of a particular intertrial interval.

The different patterns of results for food and no-food trials suggest that the pigeons' performance can be appropriately described as a strong tendency to choose the color associated with reinforcement on no-food trials unless a memory trace of food is present, in which case the other color is selected. This appears to be the only way of accounting for the fact that performance on food trials tended to fall below chance level with long retention intervals; all but one subject scored correctly on less than $50 \%$ of food trials, for both the 18 - and 22 -sec intervals. Different results for food and no-food trials have also been reported by Maki et al. (1977) and by Colwill and Dickinson (1980a); more recently, Colwill $(1982,1984 a$, 1984b) has reported below-chance performance on food trials when very long intervals are employed and has independently suggested the analysis of performance outlined above.

In conclusion, the results of this experiment indicated that the procedure is a satisfactory one for assessing a bird's memory for the recent delivery of food, so long as performance on food trials is separated from overall performance. Under the present conditions, it made no

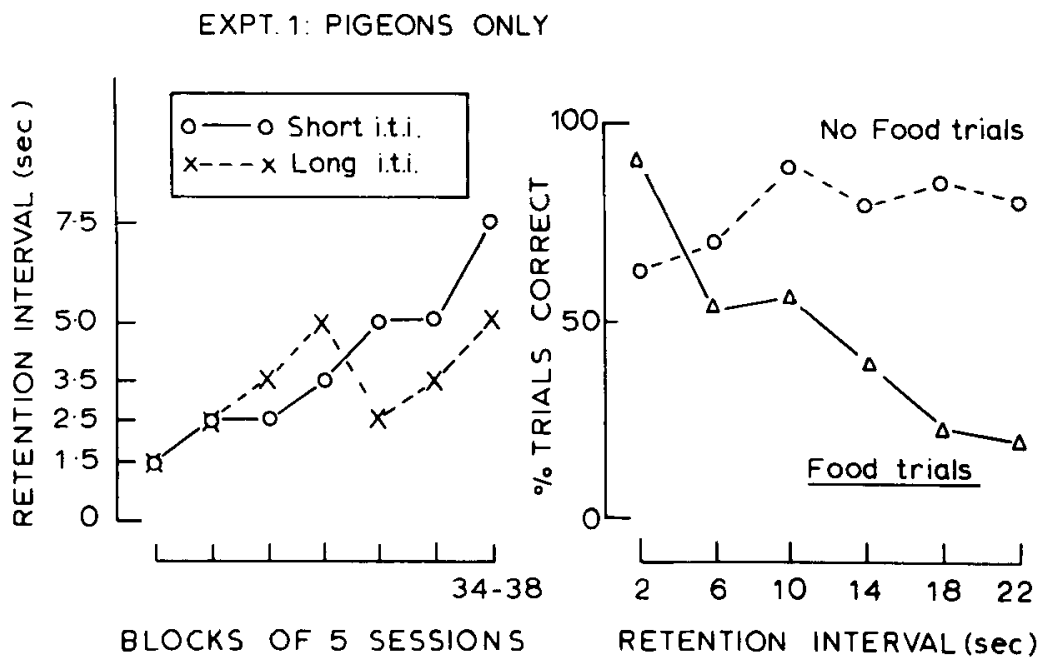

Figure 1. Experiment 1: The left-hand panel shows the median settings of the retention interval for successive blocks of five sessions during the training phase; the righthand panel shows performance during the final test sessions as percent trials correct for different retention intervals, with food and no-food trials plotted separately. 
difference whether the intertrial interval was set at $10 \mathrm{sec}$ or at $40 \mathrm{sec}$. Although the acquisition data suggested that extended training might produce good performance over longer intervals, they indicated that the rate of gain could be slight after 30 or so sessions.

\section{EXPERIMENT 2}

This experiment compared the performances of pigeons and jackdaws that were matched in terms of previous experimental history, using the general procedure employed in Experiment 1. The main changes were that we used the same intertrial interval $(20 \mathrm{sec})$ for all subjects, we gave three final test sessions, and during these three sessions, we used retention intervals equidistant on a logarithmic, rather than arithmetic, scale.

\section{Method}

Subjects. Four jackdaws, Corvus monedulus, and 8 pigeons, Columbia livia, were maintained at $85 \%$ of their free-feeding weights throughout the experiment. All 12 animals had previously served in an autoshaping experiment and then in one involving a conditional discrimination without delay (Wilson et al., 1985). As noted below, one jackdaw died during the training phase.

Apparatus. This was exactly as in Experiment 1. Reinforcement for the jackdaws consisted of $\mathbf{4 5} \mathrm{mg}$ Noyes dog food pellets.

Procedure. Following some brief manual training to ensure that each subject readily pecked at the side keys and ate the pellets, 10 sessions of training with a zero retention interval were given, as in Experiment 1. These were followed by 30 sessions in which the retention interval was increased using the adjustment procedure employed previously, resulting in intervals of $0.5,1.0,1.5 \mathrm{sec}$, and so on. Forty or more correct trials out of 60 in two successive sessions was again used as the criterion for shifting to a longer interval, one session with less than 40 correct as the criterion for shifting to a shorter interval. After 30 sessions, the value of $t$, the retention interval, was set at one step below the median value over the last 5 sessions for each subject. Each subject was required to obtain 40 or more correct trials at this setting of $t$ before the test procedure began. When a subject failed to reach this criterion, a further training session was given with the same retention interval.

The three test sessions each contained 60 trials with a fixed 20 sec intertrial interval, as in training. Six values of retention interval each occurred 10 times within each session. The values were chosen to span the range of performance already exhibited during training and were $2.0,3.6,6.5,11.7,21.0$, and $37.8 \mathrm{sec}$. In other respects the procedure was the same as in Experiment 1.

\section{Results}

During the initial 10 sessions with the zero interval condition, the performance of the pigeons was better than that of the jackdaws. Thus, on the 10th session the median percentage of correct trials for the 4 jackdaws $(67 \%)$ was reliably less than that for the 8 pigeons $(82 \%)$ [MannWhitney $\mathrm{U}(4,8)=5.5, \mathrm{p}<.05]$. However, once the retention interval was introduced, the jackdaws soon began to perform as well as the pigeons and eventually performed much better. Unfortunately, one jackdaw became seriously ill after the 10th session of this phase and died shortly afterward. Its performance up to this point had been very similar to that of the other 3 jackdaws. Taking the 3 sessions prior to the death of this bird, the median setting was $2.8 \mathrm{sec}$ for the jackdaws and $1 \mathrm{sec}$ for the pigeons. All other jackdaw data reported are from the remaining 3 birds.

Acquisition data are presented in Figure 2 in the same way that equivalent results from Experiment 1 were presented in Figure 1; for each individual, the median $t$ value for blocks of five successive sessions was obtained, and the median of these values is given for each group. It may be seen that the pigeons performed at a level comparable to that of the pigeons in the previous experiment: once $t$ values of between 5 and $10 \mathrm{sec}$ were reached, performance improved at a very slow rate, and no pigeons reached the criterion within a session in which the retention interval was set beyond $11.5 \mathrm{sec}$. In contrast, the jackdaws' performance continued to improve at a steady rate, and all 3 birds produced at least one criterion session with $\mathrm{t}$ set at $17 \mathrm{sec}$. As Figure 2 suggests, it seems likely that with a moderate extension of the training procedure the difference between the two groups would have widened even further. Mann-Whitney tests were applied to the median $t$ settings for successive blocks of five sessions and showed reliable group differences for Sessions 21-25 and Sessions $26-30$ [Us $(3,8)=3$ and 1 , respectively; $\mathrm{p}<.05$ ].

The results from the test sessions are shown in Figure 3; the left-hand panel gives the data from food trials and the right-hand panel gives the data from no-food trials. Results from the similar test in Experiment 1 have been included for purposes of comparison; it should be noted that the abscissa in Figure 3 uses a log scale and thus the functions for Experiment 1 are transformations of those shown in the right-hand panel of Figure 1. One important aspect of Figure 3 is that for both jackdaws and pigeons, length of the retention interval affected performance only on food trials; reliable trends were found on these trials for both the jackdaws $[L(3,6)=266, p<.001]$ and the pigeons

EXPT. 2: ACQUISITION DATA

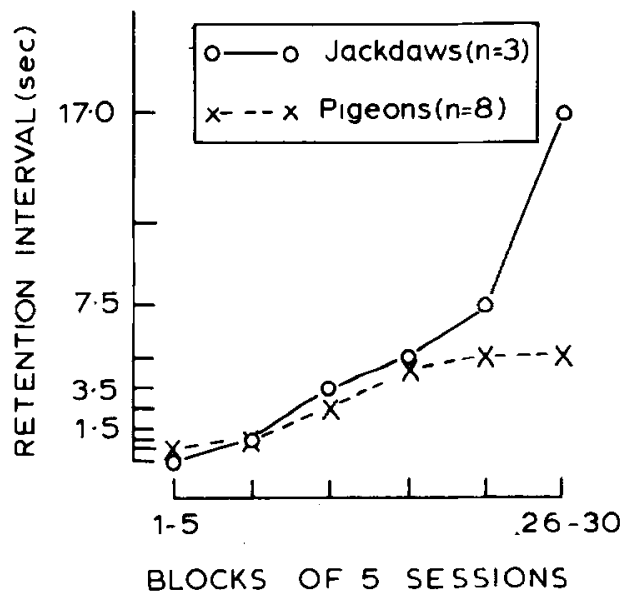

Figure 2. Experiment 2: Performance during the training phase, shown as median retention interval settings for successive blocks of five sessions. 


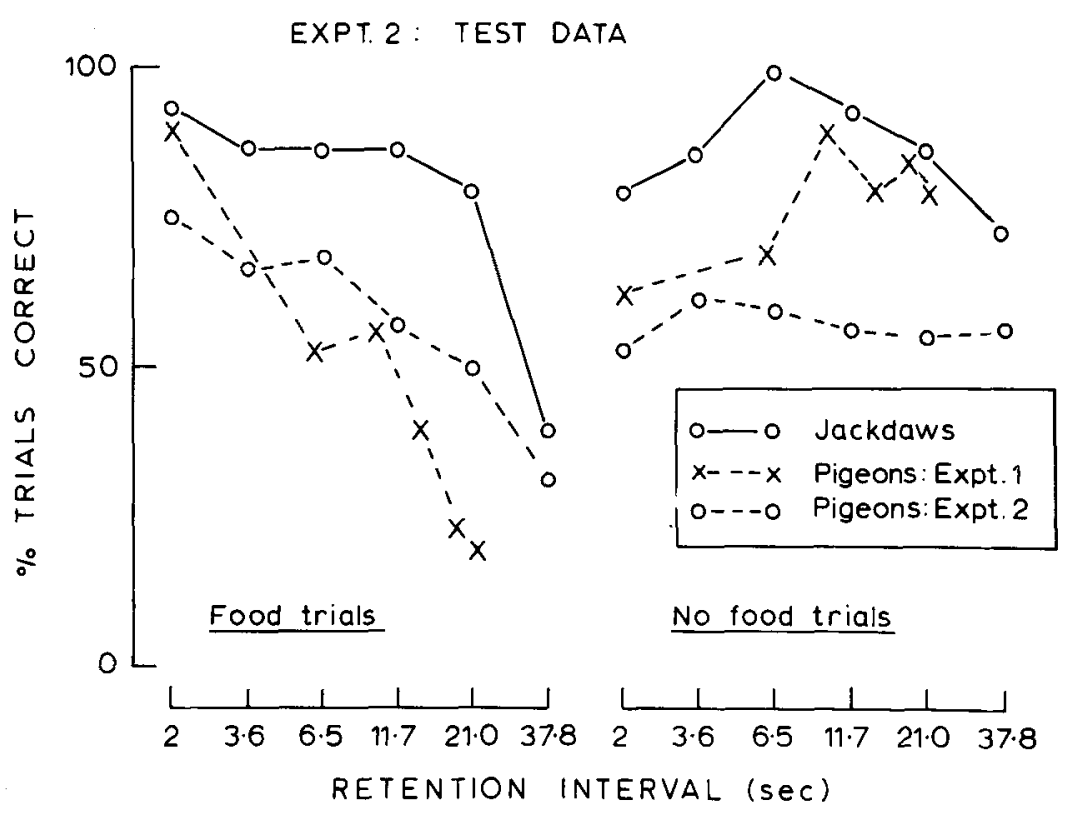

Figure 3. Results from the final tests for Experiment 2, showing percent correct trials as a function of retention interval with data from food trials in the left-hand panel and data from no-food trials in the right-hand panel. Equivalent results from Experiment 1, as shown in the right-hand panel of Figure 1, are replotted here for ease of comparison.

$[\mathrm{L}(8,6)=697, \mathrm{p}<.01]$, but no such trend was found on no-food trials in either group $(p>.05)$. Thus, the jackdaws appear to have adopted the strategy observed for the pigeons of Experiment 1 in choosing the color associated with reinforcement on no-food trials unless a memory trace of food was present.

When overall numbers of correct trials in the three test sessions were compared for the two groups, all 3 jackdaws were superior to any of the 8 pigeons $[\mathrm{U}(3,8)=$ $0, p<.01]$. When food and no-food trials were analyzed separately, jackdaws were found to score significantly higher than pigeons on no-food trials $[\mathrm{U}(3,8)=0$, $\mathrm{p}<.01]$, but the groups did not differ reliably on food trials. Examination of the results showed that on these trials 2 of the jackdaws made the greatest number of correct responses out of all subjects at all intervals except for 2.0 and $37.8 \mathrm{sec}$, but that the third jackdaw scored very well only on no-food trials; on food trials its performance was in the middle of the range for pigeons.

\section{GENERAL DISCUSSION}

Because of the small number of jackdaws employed in this study, the results need to be treated with caution. It was particularly unfortunate that one of these precious subjects was lost prior to testing in Experiment 2. Nonetheless, the overall pattern of results is compelling, and, despite the small numbers, many of the differences between pigeons and jackdaws found in the second experiment were statistically significant. Thus, both the acquisition data and the overall test results from this experi- ment point to the superiority of jackdaws in this short-term memory task. Added support for this general conclusion comes from the fact that the test performances of the pigeons in this experiment were very similar to those of the pigeons in Experiment 1, despite slight differences in procedure and large differences in previous experimental history.

We acknowledged earlier that such differences may result from the choice of a procedure that favors one species over another. Thus, with a different choice of reinforcers or a stimulus other than a white key light to signal the start of a trial, pigeons might be found to perform better than jackdaws on this kind of task. Ideally, one would need to determine separately for each species the conditions yielding optimal performance. However, this would have required resources well beyond the scope of the present research.

As noted earlier, the impetus for the present study came from the finding that corvids are superior to pigeons on a learning-set task, and from the suggestion that the basis for this might be the relative difficulty pigeons have in responding on the basis of events that occurred more than approximately $10 \mathrm{sec}$ earlier. The present results support this suggestion, in that the jackdaws were better at remembering whether food had recently been presented. However, in a discrimination learning set, subjects have to remember two events from the previous trial, namely, what stimulus was chosen as well as whether reinforcement occurred. An interesting extension of the present study would be to compare corvids with pigeons on shortterm memory for combinations of events. 


\section{REFERENCES}

Bessemer, D. W., \& STOLlNITZ, F. (1971). Retention of discriminations and an analysis of learning set. In A. M. Schrier \& F. Stollnitz (Eds.), The behavior of nonhuman primates (Vol. 4). New York: Academic.

Colwill, R. M. (1982), Associative learning and post-trial processing. Unpublished doctoral dissertation, University of Cambridge.

Colwill, R. M. (1984a). Controlled processing in pigeons. Animal Learning \& Behavior, 12, 285-291.

Colwill, R. M. (1984b). Disruption of short-term memory for reinforcement by ambient illumination. Quarterly Journal of Experimental Psychology, 36B, 235-258.

Colwill, R. M., Dickinson, A. (1980a). Short-term retention of 'surprising' events by pigeons. Quarterly Joumal of Experimental Psychology, 32, 539-556.

Colwill, R. M., \& Dickinson, A. (1980b). Short-term retention of "surprising" events following different training conditions. Animal Learning \& Behavior, 8, 561-566.

HoNIG, W. K. (1978). Studies of working memory in the pigeon. In S. H. Hulse, H. Fowler, \& W. K. Honig (Eds.), Cognitive processes in animal behavior (pp. 211-248). Hillsdale, NJ: Erlbaum.

Kamil, A. C., Jones, T. B., Pietrewicz, A., \& Mauldin, J. E. (1977). Positive transfer from successive reversal training to learning set in blue jays (Cyanocitta cristata). Journal of Comparative \& Physiological Psychology, 91, 79-86.

Kamil, A. C., Lougee, M., \& Shulman, R. I. (1973). Learning-set behavior in the learning-set experienced blue jay (Cyanocitta cristata). Journal of Comparative \& Physiological Psychology, 82, 394-405.

KAMIL, A. C., \& MaUldiN, J. E. (1975). Intraproblem retention during learning-set acquisition in bluejays (Cyanocitta cristata). Animal Learning \& Behavior, 3, 125-130.

Levine, M. (1959). A model of hypothesis behavior in discrimination learning set. Psychological Review, 66, 353-366.
Mackintosh, N. J., Wilson, B., \& Boakes, R. A. (1985). Differences in mechanisms of intelligence among vertebrates. Philosophical Transactions of the Royal Society of London, B308, 53-65.

MACPHAIL, E. M. (1982). Brain and intelligence in vertebrates. Oxford: Clarendon.

MAKI, W. S. (1981). Directed forgetting in animals. In N. E. Spear \& R. R. Miller (Eds.), Information processing in animals: Memory mechanisms. Hillsdale, NJ: Erlbaum.

MAKI, W. S., MoE, J. C., \& Bierley, C. M. (1977). Short-term memory for stimuli, responses and reinforcers. Journal of Experimental Psychology: Animal Behavior Processes, 3, 156-177.

SChrier, A. M. (1974). Transfer between repeated reversal and learningset tasks: A reexamination. Journal of Comparative \& Physiological Psychology, 87, 1004-1010.

SchUSTERMaN, R. J. (1962). Transfer effects of successive discrimination training in chimpanzees. Science, 137, 422-423.

SChUSTERMaN, R. J. (1964). Successive discrimination-reversal training and multiple discrimination training in one-trial learning by chimpanzees. Joumal of Comparative \& Physiological Psychology, 58, 153-156.

WARREN, J. M. (1966). Reversal learning and the formation of learning sets by cats and rhesus monkeys. Journal of Comparative \& Physiological Psychology, 61, 421-428.

WiLson, B. J. (1978). Complex learning in birds. Unpublished doctoral dissertation, University of Sussex, Brighton, England.

Wilson, B. J., Mackintosh, N. J., \& BoakeS, R. A. (1985). Transfer of relational rules in matching and oddity learning by pigeons and corvids. Quarterly Joumal of Experimental Psychology, 37B, 313-332.

ZEIGLER, H. P. (1961). Learning-set formation in pigeons. Journal of Comparative \& Physiological Psychology, 54, 252-254.

(Manuscript received March 12, 1985; revision accepted for publication August 22, 1985.) 